\title{
Proyecto Emily-Educación basada en problemas
}

Mónica Elizabeth Mantilla Hidalgo monica.mantilla@epn.edu.ec

Escuela Politécnica Nacional-EPN

Tamara Silvana Monar Aguiar tamara.monar@epn.edu.ec Escuela Politécnica Nacional-EPN

Adrián Santiago Aguinaga Romero asaguinaga@edu.espe.ec Universidad de la Fuerzas Armadas-ESPE

Quito-Ecuador

\section{RESUMEN}

El proyecto Emily - Educación Basada en Problemas (EBP), fue generado en la Escuela Politécnica Nacional (EPN), una institución de educación superior, como un proyecto de vinculación con la sociedad. Su propósito fue, el apoyo académico en el proceso de enseñanza-aprendizaje de la matemática, esperando que esto incidiera en el rendimiento escolar, así como también en el genuino interés por la matemática y su relación con las demás ciencias.

Los colaboradores del proyecto fueron docentes de la institución, quienes dieron seguimiento y evaluación de las actividades planificadas. Los estudiantes de la institución, quienes brindaron el apoyo académico en sesiones semanales, fueron llamados tutores. Y los beneficiarios fueron adolescentes trabajadores de la calle y su respectivo grupo familiar, acogidos por el Centro del Muchacho Trabajador (CMT).

El trabajo inició con la evaluación diagnóstica, se nivelaron los contenidos básicos. Para luego aplicar la metodología EBP relacionando distintos contenidos de matemática con otras ciencias, siempre fomentando el trabajo grupal, dando como resultado el desarrollo de habilidades y destrezas. Inicialmente se observó una resistencia a la metodología por parte de los beneficiarios, posteriormente reaccionaron favorablemente, mejorando su actitud ante la matemática y una mayor conciencia de sus propias limitaciones y capacidades.

Palabras clave: educación basada en problemas; matemática; trabajo en grupo; vinculación con la sociedad. 


\title{
Emily Project-Problem Based Learning
}

\begin{abstract}
Emily Project - Problem Based Learning (EBP), was produced by Escuela Politécnica Nacional, a higher education institution, as a project of connection with society. Its purpose was academic support in the teaching-learning process of mathematics, hoping that this would affect school performance, as well as a genuine interest in mathematics and its relationship with other sciences.

The project collaborators were teachers from the institution, who monitored and evaluated the planned activities. The students of the institution, who provided academic support in weekly sessions, were called tutors. The beneficiaries were adolescent street workers and their respective family group, hosted by the Centro del Muchacho Trabajador (CMT).

The work began with the diagnostic evaluation, and the basic knowledge were leveled. After, EBP methodology was applied relating different content of mathematics with other sciences. The project promoted group work and produced the development of abilities and skills of the beneficiaries. Initially, there was resistance from the beneficiaries to the methodology, then they reacted favorably, improving their attitude towards mathematics and a greater awareness of their own limitations and capabilities.
\end{abstract}

Keywords: Problem base learning; Mathematics; group work; connection with society.

Artículo recibido: 05 octubre. 2021 Aceptado para publicación: 02 noviembre 2021 Correspondencia: monica.mantilla@epn.edu.ec Conflictos de Interés: Ninguna que declarar 


\section{INTRODUCCIÓN}

La Educación Basada en Problemas (EBP), es un método de enseñanza que se lo comienza a aplicar en la década de los años 60 en la Escuela de Medicina de la Universidad de Case Western Reserve en los Estados Unidos y en la Universidad de McMaster en Canadá. Esto se lo hizo con el afán de mejorar la preparación de sus estudiantes y poder satisfacer las necesidades de la demanda profesional de la época.

De esta manera, se pretendía buscar un perfil más adecuado de los egresados de la carrera de medicina, en el cual se requería no sólo el conocimiento en sí, sino que adquieran las habilidades para solucionar problemas de una manera más efectiva, dentro de las cuales primaban aquellas como: adquirir información, sintetizar esta información a través del planteamiento de hipótesis y luego probar estas hipótesis para poder obtener una nueva información, o información adicional.

Luego de analizar el éxito que tuvo este proyecto, otras universidades implementaron esta metodología, y se podría decir que dentro de los treinta últimos años una diversidad de escuelas profesionales, la han incorporado en su educación superior y se sigue incrementando día a día.

Según Barrows (1986), el EBP se define como "un método de aprendizaje basado en el principio de usar problemas como punto de partida e integración de los nuevos conocimientos"; y sus principios se basan en los siguientes enunciados:

- El aprendizaje está basado en el alumno.

- El aprendizaje se produce en grupos pequeños de estudiantes.

- Los profesores son facilitadores o guías.

- Los problemas forman el foco de organización y estímulo para el aprendizaje.

- Los problemas son un vehículo para el desarrollo de habilidades de resolución de problemas clínicos.

- La nueva información se adquiere a través del aprendizaje auto dirigido.

Una vez implementado esta metodología, en el transcurso de los años se ha logrado concluir que esta metodología:

- Facilita la comprensión de los nuevos conocimientos, lo que resulta indispensable para lograr aprendizajes significativos.

- Promueve la disposición afectiva y la motivación de los estudiantes, indispensables para lograr aprendizajes significativos. 
- Provoca conflictos cognitivos en los estudiantes.

- El aprendizaje resulta fundamentalmente de la colaboración y la cooperación.

- Permite la actualización de la Zona de Desarrollo Próximo de los estudiantes.

\section{Esta metodología recoge los siguientes principios del constructivismo:}

1. El entendimiento con respecto a una situación de la realidad surge de las interacciones con el medio ambiente.

2. El conflicto cognitivo al enfrentar cada nueva situación estimula el aprendizaje.

3. El conocimiento se desarrolla mediante el reconocimiento y aceptación de los procesos sociales y de la evaluación de las diferentes interpretaciones individuales del mismo fenómeno (Fosnot, 1996).

El propósito del proyecto fue el apoyo académico efectivo en el proceso de enseñanzaaprendizaje de la matemática, una de las asignaturas más importantes en el desarrollo del pensamiento lógico, crítico y creativo, esperando que este acompañamiento incidiera en el rendimiento escolar de los beneficiarios, así como en despertar el genuino interés por la matemática y su relación con las demás ciencias.

La Educación Basada en Problemas favorece el desarrollo de habilidades en cuanto a la búsqueda y manejo de información, además, desarrolla las habilidades de investigación ya que, los estudiantes en el proceso de aprendizaje tendrán que, a partir de un enunciado, averiguar y comprender el problema y lograr una solución adecuada.

Los beneficiarios del proyecto fueron adolescentes trabajadores de la calle y su respectivo grupo familiar, acogidos por el Centro del Muchacho Trabajador (CMT), de los sectores urbano-marginales del Cantón Quito, Provincia de Pichincha. El CMT es una organización social de la Compañía de Jesús, que desarrolla una propuesta global que permita rescatar, proyectar y recrear la formación integral del niño trabajador y de su grupo familiar. Contribuyen a la formación de personas capaces de crear, desde sus propios esfuerzos, espacios personales y comunitarios de ocupación laboral y realización humana que aporten a superar su situación de extrema pobreza. Su misión es que las familias de los niños trabajadores estén unidas en su compromiso cristiano, sólidamente autofinanciadas, con acceso a educación, salud y servicios básicos de calidad; enviadas a ser agentes de cambio, orientadas a la construcción de una sociedad justa y solidaria que promueva la participación equitativa y el respeto a sus derechos fundamentales. 
Su gestión se basa en la creación, el desarrollo y el fortalecimiento de valores, mediante el cambio de actitudes y comportamientos a través de programas de formación para los niños trabajadores que ingresan al programa con todos los miembros de su grupo familiar. Este proceso de cambio gira alrededor de diez áreas importantes de la vida: lealtad, formación personal, familia, religión, educación, economía, trabajo, recreo, salud y vivienda. Estas características hacen del CMT un agente innovador de cambio social.

Los estudiantes matriculados en al menos cuarto semestre de la Escuela Politécnica Nacional, de las distintas carreras fueron los tutores, quienes brindaron el apoyo académico en sesiones semanales. En la primera etapa luego de la evaluación diagnóstica se nivelaron los contenidos básicos. En la segunda etapa se plantearon problemas integradores que relacionen distintos contenidos de matemática con otras ciencias para favorecer el razonamiento y juicio crítico,

Los docentes del DFB de la EPN actuarán como profesores colaboradores, los mismos que serán los responsables de la capacitación a los tutores y de dar seguimiento y evaluación del cumplimiento de las actividades y tareas encomendadas.

\section{ESTRATEGIAS METODOLÓGICAS O MATERIALES Y MÉTODOS}

En las últimas décadas se han evidenciado grandes cambios producidos en todos los ámbitos de nuestra cotidianidad debido al avance de la tecnología y el acceso a la información. Esto modifica el entorno y las estructuras mentales con la que crecen las actuales generaciones, razón por la cual la modalidad de enseñanza focalizada hacia los contenidos, priorizando los conceptos abstractos sobre los ejemplos concretos y las aplicaciones no alcanza a cumplir con los objetivos planteados. Además, las técnicas de evaluación se limitan a comprobar la memorización de información y de hechos, ocupándose muy rara vez de desafiar al estudiante para alcanzar aprendizajes especialmente relacionados con el razonamiento y el juicio crítico.

Por otro lado, los estudiantes en un cierto momento van presentando una cierta ansiedad o miedo a contenidos que involucran la matemática, por tanto, la enseñanza de la matemática debe estar basada en quitarle fuerza justamente a este problema, para lo cual muchos investigadores recomiendan diseñar situaciones de aprender, de cómo construir mentalmente eso que se va a aplicar después. Debemos incursionar en el arte de diseñar situaciones de aprendizaje, problemas integrados en donde aparece la matemática, situaciones problemas. 
En el Ecuador el sistema de educación a todo nivel no contempla la importancia de este problema ni de generar un interés genuino por el estudio de la matemática y su relación con las demás ciencias. Parecería que cada ámbito del conocimiento no se relaciona con los demás, lo que conlleva la percepción de una falta de utilidad de los contenidos matemáticos del currículo.

Los escasos recursos económicos de las familias más desfavorecidas no son suficientes para contemplar la posibilidad de un profesor particular o un curso remedial, para mejorar su desempeño académico. Todo esto desencadena en uno de los factores de baja autoestima y en desmotivación, que finalmente, es una de las causas de deserción y de repetición de los cursos de la educación formal.

El presente proyecto pretende potenciar el desarrollo integral (conocimientos, procedimientos, habilidades, actitudes y valores) a este sector de estudiantes ofreciéndoles un apoyo académico que mejore su rendimiento en general y esto les motive a culminar sus estudios y aún más a pensar en su futura formación profesional.

Para cumplir con el objetivo planteado; potenciar el desarrollo integral del estudiante de educación general básica y del bachillerato general unificado, fomentando una actitud positiva hacia su propio aprendizaje, mediante la aplicación de la educación basada en problemas, se planificaron actividades, tales como:

- Talleres para los profesores colaboradores para profundizar en la metodología de Educación Basada en Problemas, planificación de los contenidos, tomando como guía los textos del Ministerio de Educación vigentes.

- Capacitación a los estudiantes de la EPN quienes actuaron como tutores, seguimiento y evaluación de sus responsabilidades en cada sesión.

- Reuniones semanales entre profesores colaboradores y tutores para el planteamiento de los problemas integradores que serían planteados cada sábado en las reuniones de trabajo con los beneficiarios, llamada Guía de clase.

- Proceso de inscripción, registro y asignación de beneficiarios a los diferentes grupos de trabajo.

- Evaluación diagnóstica a los estudiantes beneficiarios, para conocer el nivel de conocimiento, debilidades, destrezas y habilidades.

- Evaluación continua del trabajo colaborativo, la disciplina, puntualidad, actitud y aptitud durante cada sesión de trabajo, en los respectivos grupos. 
- Reuniones periódicas con autoridades y docentes del Centro del Muchacho Trabajador para planificar modificaciones necesarias en los contenidos revisados, en los tiempos de dedicación, en las estrategias de motivación para lograr mantener a la mayor parte de beneficiarios en todo el proceso.

\section{RESULTADOS Y DISCUSIÓN}

El proyecto no logró desarrollarse en un $100 \%$, debido a varios factores no considerados en su planificación, algunos de los cuales se indican a continuación:

1. El año lectivo inicia en los primeros días de septiembre y el proyecto empezó en enero, lo que produjo un desfase.

2. Los tutores eran seleccionados y capacitados durante el interciclo (por la disponibilidad de tiempo de estos), y colaboraban hasta el término del semestre, por lo que nuevos tutores debían ser seleccionados para el siguiente semestre y de esta manera concluir el año lectivo completo.

3. Debido a lo explicado en los numerales anteriores, la duración del proyecto se prolongó y se proyectó que concluya en junio del 2020. Sin embargo, la emergencia sanitaria provocada por la pandemia del COVID-19 en marzo 2020 imposibilitó el concluir el proceso.

Los tutores aprobaron el curso de Educación Basada en Problemas, dirigido por un instructor calificado, con una duración de 20 horas, de las cuales 4 fueron presenciales y las demás virtuales, cumpliendo con la asistencia y calificación mínima previas a la obtención del certificado. Del número total de participantes, el 90\% aprobó el curso. Los tutores cumplieron, en un $88 \%$, con la asistencia y participación en las reuniones con los profesores colaboradores para la planificación de actividades semanales.

En la primera reunión con los beneficiarios se realizó la evaluación diagnóstica, obteniéndose resultados desalentadores, ya que más del $70 \%$ no contestó a ninguna pregunta, lo que indicó que su asistencia no respondía a una motivación propia. Por esta razón, se mantuvo una reunión con las autoridades del CMT y se verificó que ellos habían obligado a los representantes de los beneficiadores a llevar a los beneficiarios cada sábado. Llegando a un acuerdo de que la asistencia debía ser voluntaria y resaltando las ventajas de su participación se decidió asignar las primeras 8 reuniones para realizar una primera etapa de nivelación de conocimientos, explorando en cada reunión los contenidos más relevantes. 
En las primeras reuniones semanales de trabajo se evidenció que algunos beneficiarios se quedaban en pasillos, baños, gradas e ingresaban tarde o no ingresaban a su respectivo lugar asignado. Y durante las clases, se observaron tres tipos de comportamiento:

- Agresivo, llegando en ciertos casos a antisocial

- Pasivo, pudiendo presentar algún grado de apatía

- Asertivo, demostrando interés por aprender y colaborar

En el transcurso de las semanas, la relación entre tutores y beneficiarios mejoró, lo que produjo un cambio de actitud hacia las actividades del proyecto.

Las reuniones de trabajo mantenidas con las autoridades y docentes del Centro del Muchacho Trabajador (CMT), permitieron una mejor coordinación. Sin embargo, fue necesario solicitar el retiro de tres beneficiarios, ya que presentaban problemas de comportamiento que requerían de la intervención de psicólogos.

Otro inconveniente fue que, en un principio, los problemas eran planteados al grupo y el tiempo de dedicación para terminarlo era muy extenso, por lo que las partes pendientes quedaban para la siguiente sesión de trabajo semanal e imposibilitando realizar la evaluación al final de la reunión. Esto tuvo que corregirse en la marcha haciendo las modificaciones pertinentes.

Por otra parte, se experimentó la resistencia de los beneficiarios a trabajar en grupos cuya configuración cambiaba en cada reunión de trabajo. Algunos beneficiarios mostraban resistencia a trabajar con compañeros diferentes en cada sesión. Tanto los colaboradores como los beneficiarios fueron ganando poco a poco la confianza para manejar sus grupos y para resolver las tareas lo cual permitió mejorar la actuación de todas las partes involucradas

Se analizaron los informes semanales de las evaluaciones grupales e individuales para medir el nivel de comprensión de los temas incluidos. Esta tarea fue realizada conjuntamente por los tutores y profesores colaboradores de cada grupo.

Para medir la satisfacción y la aceptación del método EBP se realizaron tres encuestas, en los últimos 3 meses de trabajo y se evaluó los siguientes parámetros:

- Relación alumno-tutor

- Interés por los temas impartidos

- Materiales y metodología usados 
- Las evaluaciones

Las preguntas aplicadas fueron evaluadas entre el rango de 0 y 5 considerando al 0 como una deficiente o mala aplicación, y el 5 como una satisfacción o conformidad positiva. Los resultados obtenidos son:

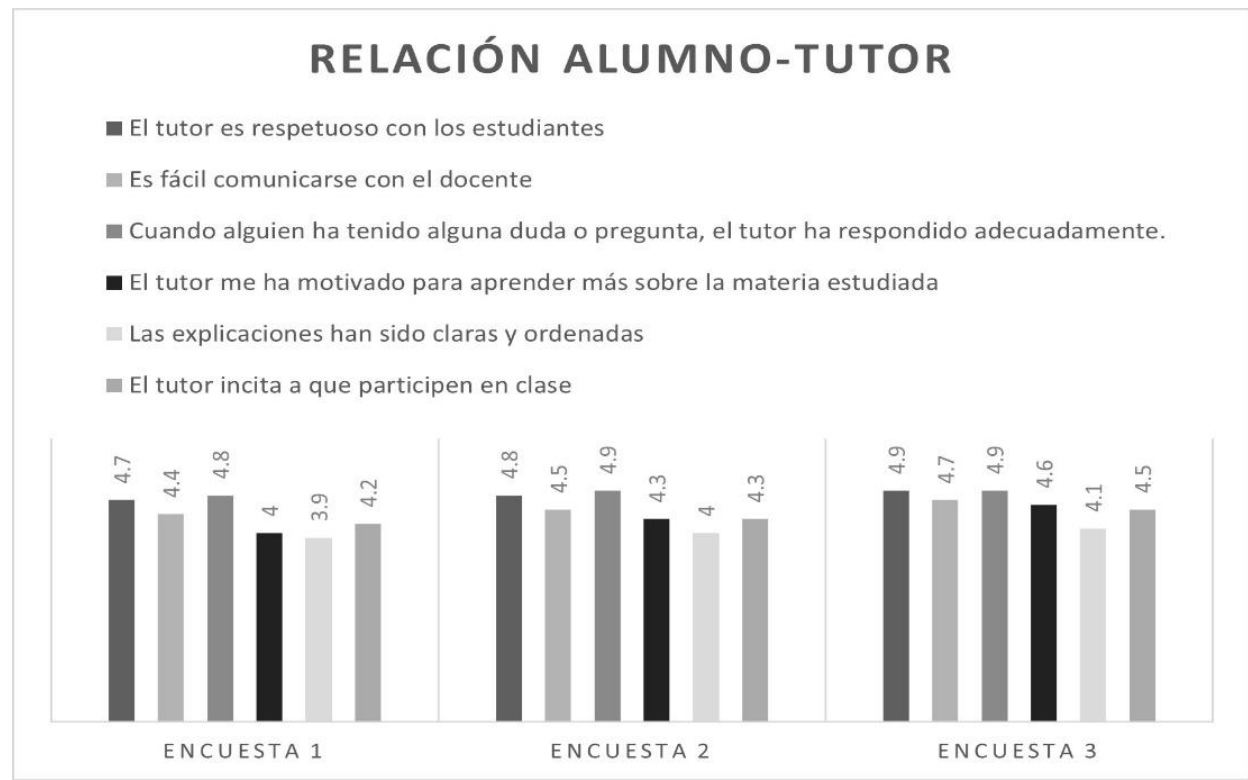

Figura 1. Resultado de las encuestas con respecto a la relación alumno-tutor.

De la figura anterior se observa que los resultados obtenidos son favorables pues están en el rango entre 3.9 a 4.9, lo que representa una buena aceptación de los tutores y una relación adecuada con los beneficiarios.

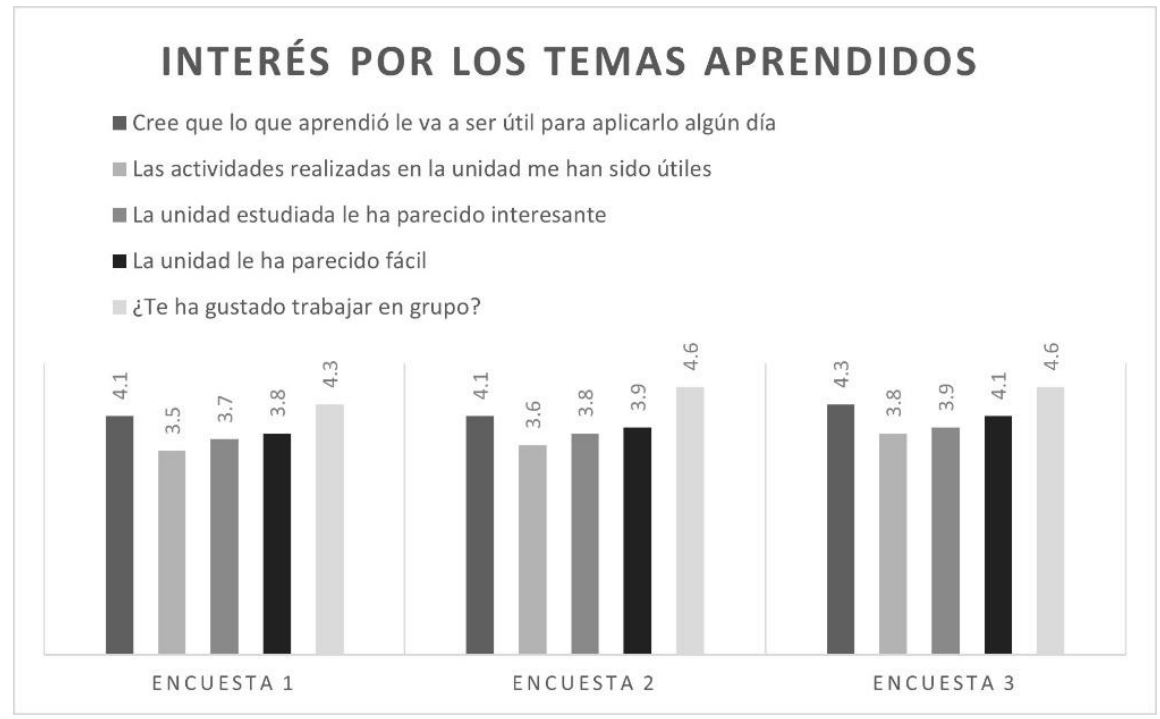

Figura 2. Resultado de las encuestas con respecto al interés de los beneficiarios en los temas de clase. 
En esta figura con respecto al interés de los estudiantes en el tema y la aplicabilidad de este, se observa una menor calificación entre 3.5 a 4.6. Estos resultados están directamente relacionados con la madurez de los beneficiarios quienes (debido a su corta edad) aún no tienen interés profundo de aprender y de visualizar la aplicación de los temas presentados en su diario vivir.

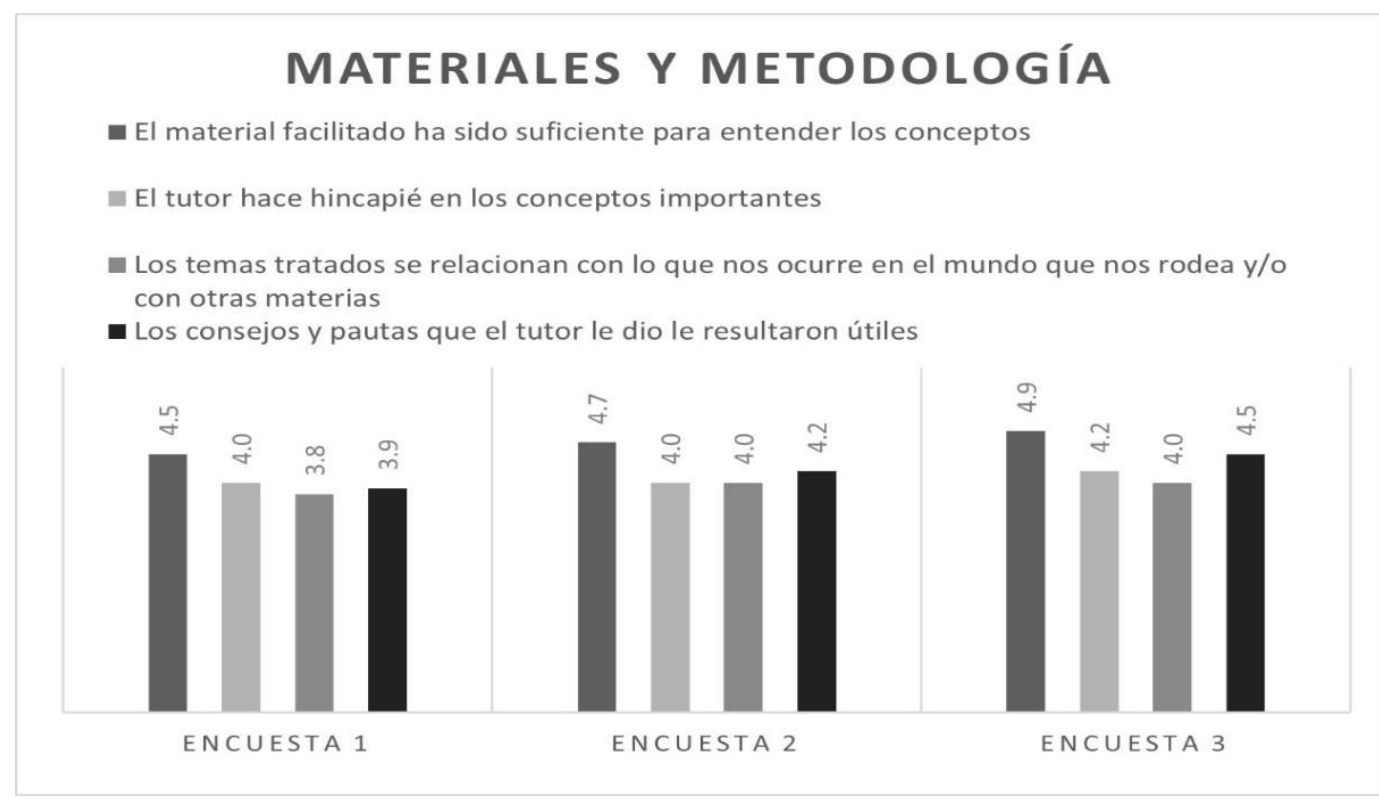

Figura 3 . Resultado de las encuestas con respecto a los materiales y metodología utilizado.

En la figura 3, con respecto a los materiales proporcionados y la metodología se puede evidenciar que hay una buena aceptación de la metodología y que los materiales proporcionados poco les facilita la comprensión.

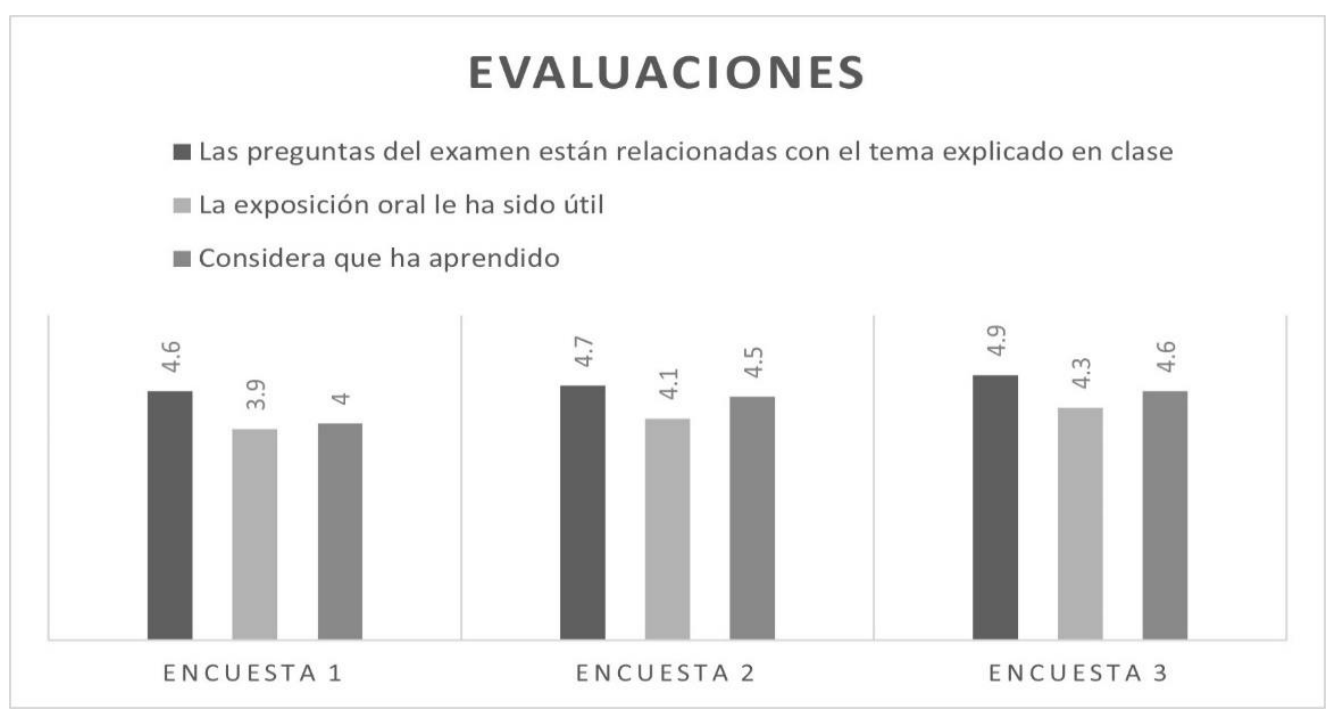

Figura 4. Resultado de las encuestas con respecto a las evaluaciones realizadas. 
En cuanto a las evaluaciones se evidenció una buena aceptación del trabajo de los tutores y colaboradores. Las calificaciones obtenidas pertenecían al rango entre 3.9 y 4.9 puntos, categorizadas como bueno y muy bueno.

\section{CONCLUSIÓN O CONSIDERACIONES FINALES}

A partir del análisis de problemáticas y la contextualización de la teoría relacionada al presente trabajo de aplicación de la educación basada en problemas, podemos concluir que:

1. Desde el inicio el proyecto tuvo gran acogida por parte de los estudiantes de la EPN que participaron como tutores y causo un impacto positivo en los beneficiarios. Lastimosamente el proyecto no logró desarrollarse al 100\%, debido a varios factores no considerados en su planificación.

2. Las dificultades iniciales con respecto a la actitud de los beneficiarios principalmente por falta de motivación y la obligatoriedad de asistencia fueron resueltas en conjunto con las autoridades y docentes del Centro del Muchacho Trabajador.

3. Los tutores se capacitaron continuamente en la metodología y contaron con el apoyo constante de los colaboradores.

4. La formulación de los problemas integradores y preparación de los contenidos pertinentes requirió de un tiempo mucho mayor al planificado, ya que tanto profesores colaboradores como tutores no habían experimentado esta metodología en sus procesos educativos.

5. En la aplicación de la metodología se evidenció que las actividades planteadas tomaban más tiempo del esperado y se dificultaba la evaluación continua, en atención a este inconveniente se realizaron las modificaciones necesarias.

6. La educación basada en problemas se nutre del trabajo en grupo y así fue planificada, pero se constató una fuerte resistencia de los beneficiarios a trabajar en grupos y peor aún, a cambiar de grupos. Por esta razón, en principio se debió permitir que se formaran los grupos a gusto de los beneficiarios, para luego poco a poco ir modificando esta situación.

7. A los tutores les tomó un tiempo adaptarse a su papel, mantener la disciplina de la clase, el manejo de los tiempos de dedicación a cada actividad y la aplicación de estrategias para apoyar en el aprendizaje, pero no dar la clase magistral o resolver los 
ejercicios por los beneficiarios, sino que los beneficiarios se involucren y cumplan las tareas asignadas como retos semana a semana.

8. La metodología aplicada entregó buenos resultados en temas cognitivos con calificaciones entre 3.5 y 5 puntos categorizadas como bueno y muy bueno. En cuanto al comportamiento, el $80 \%$ de los beneficiarios cambiaron su actitud respecto al trabajo colaborativo y al grado de compromiso con su aprendizaje.

9. Las reuniones de trabajo mantenidas con las autoridades y docentes del Centro del Muchacho Trabajador (CMT), permitieron coordinar de mejor manera tanto las actividades, contenidos y estrategias de acercamiento para favorecer el cumplimiento de objetivos.

\section{RECONOCIMIENTO}

El Centro del Muchacho Trabajador fue uno de nuestros más grandes aliados para poder desarrollar este proyecto. De acuerdo a su página de presentación se puede observar que: "Es una organización basada en valores, que busca el crecimiento del ser humano en su más íntima esencia y, desde allí, la superación de la marginalidad y la pobreza como una instancia de apretura y búsqueda de oportunidades, de educación y cohesión familiar, de dignidad, de exigibilidad de derechos y de concreción de una auténtica ciudadanía. Tiene a la persona como sujeto de la acción, trabajando con ella en el lugar en el que está, partiendo de su realidad y cultura", y está dirigida por Jesuitas. http://www.jesuitas.ec/centro-muchacho-trabajador/

\section{LISTA DE REFERENCIAS}

Barrows H.S. (1986) A Taxonomy of problem-based learning methods. Medical Education, 20: 481-486.

Morales, P. (2018). Aprendizaje basado en problemas (ABP) y habilidades de pensamiento crítico ¿una relación vinculante? Revista Electrónica Interuniversitaria de Formación del Profesorado, 21(2), 91-108. https://doi.org/10.6018/reifop.21.2.323371

Escribano, A., \& Del Valle, A. (2015). El aprendizaje basado en problemas (ABP). Bogotá: Ediciones de la U.

Esquer, F. G., Martínez, I. R., Romero, F. M., \& Valero, P. B. (2009). Aplicación interdisciplinar del aprendizaje basado en problemas en ciencias de la salud: una 
herramienta útil para el desarrollo de competencias profesionales. Revista de docencia universitaria. Vol.7 (4).

Gómez, B. R. (2005). Aprendizaje basado en problemas (ABP): una innovación didáctica para la enseñanza universitaria. Educación y educadores, Revista de docencia universitaria. (8), 9-20.

Guitart, M. E. (2011). Del “Aprendizaje Basado en Problemas” (ABP) al “Aprendizaje Basado en la Acción" (ABA). Claves para su complementariedad e implementación. REDU. Revista de docencia universitaria, 9(1), 91.

Labra, P., Kokaly, M. E., Iturra, C., Concha, A., Sasso, P., \& Vergara, M. I. (2011). El enfoque ABP en la formación inicial docente de la Universidad de Atacama: el impacto en el quehacer docente. Estudios pedagógicos (Valdivia), 37(1), 167-185.

Muñoz-Repiso, A. G. V., \& Gómez-Pablos, V. B. (2017). Aprendizaje Basado en Proyectos (ABP): evaluación desde la perspectiva de alumnos de Educación Primaria. Revista de Investigación Educativa, 35(1), 113-131.

Luy-Montejo, C. (2019). El Aprendizaje Basado en Problemas (ABP) en el desarrollo de la inteligencia emocional de estudiantes universitarios. Propósitos y representaciones, 7(2), 353-383.

Stepien W.J. (1993) Problem-based Learning: As Authentic as It Gets. Educational Leadership 50, No. 7:25-28. http://www.redalyc.org/articulo.oa?id=29901314

García, C. (2018) Metodología ABP en las clases de Matemáticas de la ESO [Tesis de Máster Universitario]. Universidad de Valladolid.

Palacios, C. (2016) El Aprendizaje basado en proyectos desde el aula de Matemáticas. Publicado en el XVI. Congreso de enseñanza y aprendizaje de las matemáticas. Cádiz -España.

Obando, G y Muñera, J. (2003). Las situaciones problemas como estrategia para la conceptualización matemática. En: Revista Educación y Pedagogía. Medellín: Universidad de Antioquia, Facultad de Educación. Vol. XV, no. 35, (enero- abril), pp. $185-199$.

Alzate, E. Montes, J, Escobar, R. (2013). Diseño de actividades mediante la metodología ABP para la enseñanza de la Matemática. Publicado en: Scientia et Technica Año XVIII, Vol 18, No.3. Universidad Tecnológica de Pereira. 
Paredes, H. D. H., Gutiérrez, E. A. M., López, J., \& Giraldo, L. E. P. (2015). Aprendizaje basado en problemas como potencializador del pensamiento matemático. Plumilla Educativa, 15(1), 299-312.

Castillo, S. (2008). Propuesta pedagógica basada en el constructivismo para el uso óptimo de las TIC en la enseñanza y el aprendizaje de la matemática. Revista latinoamericana de investigación en matemática educativa, 11(2), 171-194.

Roig-Vila, R. (2018). El compromiso académico y social a través de la investigación e innovación educativas en la Enseñanza Superior.

Semanate Zapata, R. D. (2020). Entorno Virtual de Aprendizaje de gestión académica para fortalecer el proceso de enseñanza aprendizaje de matemática a segundo año de bachillerato general unificado en la Unidad Educativa "Louis V. de Broglie" (Master's thesis, Quito).

Encalada Díaz, I. A., \& Delgado Alva, R. (2018). El uso del software educativo cuadernia en el proceso de enseñanza-aprendizaje y en el rendimiento académico de la matemática de los estudiantes del 5to año de secundaria de la institución educativa $\mathrm{N}^{\circ} 5143$ escuela de talentos Callao 2015.

Albornoz-Acosta, J. A., Maldonado-Cid, J. G., Vidal-Silva, C. L., \& Madariaga, E. (2020). Impacto y recomendaciones de clase invertida en el proceso de enseñanzaaprendizaje de geometría. Formación universitaria, 13(3), 3-10.

Gutiérrez, G., \& Berciano, A. (2012). Un experimento de enseñanza sobre la influencia del ABP en la competencia matemática con futuras maestras de Educación Infantil.

Tallart-Fabré, J., \& Guilarte-Columbié, H. (2016). Forma didáctica del aprendizaje basado en problemas en la formación inicial matemática del maestro primario en la Universidad de Oriente. Maestro y Sociedad, 13(2), 212-226.

Ardila, O. (2011). Diseño e implementación de objetos virtuales de aprendizaje como estrategia de apoyo para la enseñanza de las matemáticas en la educación técnica profesional (Doctoral dissertation, Universidad Minuto de Dios). 